\title{
Generalizations of some integrals over the unitary group
}

\author{
B. Schlittgen ${ }^{1}$ and T. Wettig ${ }^{1,2}$ \\ ${ }^{1}$ Department of Physics, Yale University, New Haven, CT 06520-8120, USA \\ 2 RIKEN-BNL Research Center, Upton, NY, 11973-5000, USA
}

\begin{abstract}
Using the character expansion method, we generalize several wellknown integrals over the unitary group to the case where general complex matrices appear in the integrand. These integrals are of interest in the theory of random matrices and may also find applications in lattice gauge theory.

PACS numbers: 11.15.Ha, 02.20.Qs
\end{abstract}

\section{Introduction}

Physical systems described by nonhermitian operators have recently attracted a lot of attention in the literature. Applications range from flux-line pinning in superconductors [1] over dissipation and scattering in quantum chaos [2] to quantum chromodynamics (QCD) at nonzero density [3]. In turn, the interest in these applications has stimulated new mathematical studies of nonhermitian matrices, in particular in the field of random matrix theory.

We have been led to consider nonhermitian matrices in our work on the color-flavor transformation [4, 5, 6]. This transformation involves an integration over complex matrices $Z$ without any symmetry requirements. Applying the transformation to lattice QCD [6, 7, 8] results in a complex action that is not amenable to standard Monte-Carlo algorithms. A possible way of trying to resolve this problem is to write $Z=H U$ with $H$ hermitian and $U$ unitary, and to perform the integral over $U$ analytically so that only the integration over $H$ has to be done numerically. This approach leads to the following integral over the unitary group,

$$
\mathcal{I}_{1}=\int_{\mathrm{U}(N)} d \mu(U) \operatorname{det}^{\nu} U e^{\frac{1}{2} \operatorname{tr}\left(A U+B U^{\dagger}\right)},
$$

where $d \mu(U)$ is the Haar measure of $\mathrm{U}(N), \nu$ is an integer, which without loss of generality we take to be non-negative, and $A, B \in \operatorname{Gl}(N, \mathbb{C})$. The above integral is the main focus of this paper. It is well-known for $A=B^{\dagger}$ [9, 10, 11] (where it leads to the effective partition function of QCD in the Leutwyler-Smilga regime [12, 10]), but to the best of our knowledge $\mathcal{I}_{1}$ had not been computed before for $A \neq B^{\dagger}$. We found that the latter case can be solved rather easily using the character expansion method put forward by Balantekin [13, 14]. Although the case of non-integer $\nu$ appears to be beyond this approach, we expect our result to hold in that case as well. Employing the same method, we could also compute the integral which arises from the factorization 
$Z=U \Lambda V^{\dagger}$ in the color-flavor transformed action mentioned above. The matrices $U$ and $V$ are unitary, while $\Lambda$ is diagonal with nonnegative entries. The resulting integral in slightly generalized form is given by

$$
\mathcal{I}_{2}=\int_{\mathrm{U}(N)} d \mu(U) \int_{\mathrm{U}(N)} d \mu(V) \operatorname{det}^{\nu}(U V) e^{\frac{1}{2} \operatorname{tr}\left(U A V B+C V^{\dagger} D U^{\dagger}\right)},
$$

where $A, B, C, D \in \operatorname{Gl}(N, \mathbb{C})$. This integral was previously known only for the case of $\nu=0, C=B^{\dagger}$ and $D=A^{\dagger}$ 15, 16, 10]. We also show that the generalization of $\mathcal{I}_{2}$ to the case of unequal dimensions of $U$ and $V$ leads to an integral which can be non-zero only if determinants of $U$ and $V$ are absent from the integrand. We conjecture an expression for the result in this case.

In Sec. 2, we derive results for $\mathcal{I}_{1}$ and $\mathcal{I}_{2}$. In addition, we discuss $\mathcal{I}_{2}$ with unequal dimensions of $U$ and $V$ and briefly consider the (trivial) generalization of the ItzyksonZuber integral to the case of general complex matrices in the integrand. We also comment on the applicability of our results to the case of noninvertible matrices. Conclusions are drawn in Sec. 3.

\section{Calculation of the group integrals}

Our calculations rely on the formalism of Ref. 14 and on several examples worked out in detail therein. Rather than reproducing the material from that work, we will refer to the relevant equations in Ref. [14] whenever appropriate. Thus, the reader is advised to have a copy of this reference at hand.

The key observation for the generalization of the results of Ref. [14] is that the representation theories of the groups $\mathrm{U}(N)$ and $\operatorname{Gl}(N)$ are essentially the same. In particular, Weyl's character formula

$$
\chi_{r}(X)=\frac{\operatorname{det}\left(x_{i}^{n_{j}+N-j}\right)}{\Delta\left(x_{1}, \ldots, x_{N}\right)},
$$

holds not just for unitary, but also for general linear matrices, cf. Ref. [17]. Here, $r=\left(n_{1}, \ldots, n_{N}\right)$ denotes an irreducible representation of $\operatorname{Gl}(N)$ labelled by nonnegative, non-increasing integers. The $x_{i}(i=1, \ldots, N)$ are the eigenvalues of the matrix $X$.

\subsection{Calculation of $\mathcal{I}_{1}$}

Using Eq. (3.5) of Ref. [14], we write

$$
\begin{aligned}
& \operatorname{det}^{\nu}(A U) e^{\operatorname{tr} A U}=\sum_{r} \alpha_{r}^{(\nu)} \chi_{r}(A U), \\
& e^{\operatorname{tr} B U^{\dagger}}=\sum_{r} \alpha_{r}^{(0)} \chi_{r}\left(B U^{\dagger}\right) .
\end{aligned}
$$

Here, the sums are over all irreducible representations of $\operatorname{Gl}(N)$ labeled by $r=$ $\left(n_{1}, n_{2}, \ldots, n_{N}\right)$ as above. The corresponding characters are denoted by $\chi_{r}$. For

convenience, we have left out the factor of $\frac{1}{2}$ in the exponent of Eq. (1), which will be reinstated in the final result. The coefficients in the character expansion are given by

$$
\alpha_{r}^{(\nu)}=\operatorname{det}\left[\frac{1}{\left(n_{j}-\nu+i-j\right) !}\right]
$$


where $i$ and $j$ run from 1 to $N$, labelling the rows and columns of the matrix. We thus obtain

$$
\begin{aligned}
\hat{\mathcal{I}}_{1} & \equiv \int_{\mathrm{U}(N)} d \mu(U) \operatorname{det}^{\nu}(A U) e^{\operatorname{tr}\left(A U+B U^{\dagger}\right)} \\
& =\sum_{r} \sum_{r^{\prime}} \alpha_{r}^{(\nu)} \alpha_{r^{\prime}}^{(0)} \int_{\mathrm{U}(N)} d \mu(U) \chi_{r}(A U) \chi_{r^{\prime}}\left(B U^{\dagger}\right) .
\end{aligned}
$$

Now, we can write

$$
\chi_{r}(A U) \chi_{r^{\prime}}\left(B U^{\dagger}\right)=A_{a b}^{(r)} U_{b a}^{(r)} B_{c d}^{\left(r^{\prime}\right)} U_{c d}^{\left(r^{\prime}\right) *},
$$

where the superscript serves as a reminder that these matrices live in the representations $r$ and $r^{\prime}$ of $\mathrm{Gl}(N)$, respectively. If we restrict ourselves to unitary matrices, we have corresponding irreducible representations of the subgroup $\mathrm{U}(N)$ of $\mathrm{Gl}(N)$, and hence, we can use the group theoretical result

$$
\int_{\mathrm{U}(N)} d \mu(U) U_{b a}^{(r)} U_{c d}^{\left(r^{\prime}\right) *}=\frac{1}{d_{r}} \delta^{r r^{\prime}} \delta_{b c} \delta_{a d},
$$

where $d_{r}$ is the dimension of $r$, regardless of whether we consider it as a representation of $\mathrm{Gl}(N)$ or $\mathrm{U}(N)$. It then follows that

$$
\hat{\mathcal{I}}_{1}=\sum_{r} \frac{\alpha_{r}^{(0)}}{d_{r}} \alpha_{r}^{(\nu)} \chi_{r}(A B) .
$$

From Eqs. (3.5), (3.3) and (2.9) of Ref. [14], we obtain

$$
\frac{\alpha_{r}^{(\nu)}}{d_{r}}=\prod_{i=1}^{N} \frac{(N-i) !}{\left(k_{i}-\nu\right) !} \quad \text { with } k_{i}=N+n_{i}-i
$$

The matrix $A B$ has $N$ eigenvalues which we denote by $\mu_{1}^{2}, \ldots, \mu_{N}^{2}$. We now apply Weyl's formula,

$$
\chi_{r}(A B)=\frac{\operatorname{det}\left[\mu_{i}^{2\left(n_{j}+N-j\right)}\right]}{\Delta\left(\mu^{2}\right)},
$$

where

$$
\Delta(x)=\prod_{i<j}^{N}\left(x_{i}-x_{j}\right)
$$

is the Vandermonde determinant. Inserting this expression, together with Eqs. (6) and (11), into Eq. (10) yields

$$
\hat{\mathcal{I}}_{1}=\left[\prod_{n=1}^{N-1} n !\right] \frac{1}{\Delta\left(\mu^{2}\right)} \sum_{r} \operatorname{det}\left[\frac{1}{k_{j} !\left(k_{j}-N-\nu+i\right) !}\right] \operatorname{det}\left[\mu_{i}^{2 k_{j}}\right] .
$$

Applying the Binet-Cauchy formula, see Eq. (B4) of Ref. [14], and using the power series expansion of the Bessel function,

$$
\frac{I_{\lambda}(2 y)}{y^{\lambda}}=\sum_{k=0}^{\infty} \frac{y^{2 k}}{k !(k+\lambda) !}
$$


we obtain immediately

$$
\hat{\mathcal{I}}_{1}=\left[\prod_{n=1}^{N-1} n !\right] \frac{1}{\Delta\left(\mu^{2}\right)} \operatorname{det}\left[\mu_{j}^{N-i+\nu} I_{i-N-\nu}\left(2 \mu_{j}\right)\right] .
$$

Pulling out the factors of $\mu_{j}^{\nu}$ and rearranging the determinant using $I_{n}=I_{-n}$ yields

$$
\begin{aligned}
\operatorname{det}\left[\mu_{j}^{N-i+\nu} I_{i-N-\nu}\left(2 \mu_{j}\right)\right] & =\operatorname{det}^{\frac{\nu}{2}}(A B) \operatorname{det}\left[\mu_{j}^{N-i} I_{i-N-\nu}\left(2 \mu_{j}\right)\right] \\
& =\operatorname{det}^{\frac{\nu}{2}}(A B) \operatorname{det}\left[\mu_{j}^{i-1} I_{\nu+i-1}\left(2 \mu_{j}\right)\right] .
\end{aligned}
$$

Finally, we rescale $A$ and $B$ by $\frac{1}{2}$ to reinstate the factor of $\frac{1}{2}$ in the exponent of Eq. (1) to obtain

$$
\begin{aligned}
\int_{\mathrm{U}(N)} d \mu(U) \operatorname{det}^{\nu} U e^{\frac{1}{2} \operatorname{tr}\left(A U+B U^{\dagger}\right)} \\
=2^{\frac{N(N-1)}{2}}\left[\prod_{n=1}^{N-1} n !\right]\left(\frac{\operatorname{det} B}{\operatorname{det} A}\right)^{\frac{\nu}{2}} \frac{\operatorname{det}\left[\mu_{i}^{j-1} I_{\nu+j-1}\left(\mu_{i}\right)\right]}{\Delta\left(\mu^{2}\right)},
\end{aligned}
$$

where, once again, the $\mu_{i}^{2}$ are the eigenvalues of $A B$.

\subsection{Calculation of $\mathcal{I}_{2}$}

Note first that in the integrand of $\mathcal{I}_{2}$, the determinants of $U$ and $V$ have to be raised to the same power, as indicated in Eq. (2); otherwise the integrations over the U(1) subgroups of $U$ and $V$ simply render $\mathcal{I}_{2}$ zero.

Using again Eq. (3.5) of Ref. [14], we have

$$
\begin{aligned}
& \operatorname{det}^{\nu}(U A V B) e^{\operatorname{tr} U A V B}=\sum_{r} \alpha_{r}^{(\nu)} \chi_{r}(A U V B), \\
& e^{\operatorname{tr} C V^{\dagger} D U^{\dagger}}=\sum_{r} \alpha_{r}^{(0)} \chi_{r}\left(C V^{\dagger} D U^{\dagger}\right)
\end{aligned}
$$

with $\alpha_{r}^{(\nu)}$ given in Eq. (6). Thus,

$$
\begin{aligned}
\hat{\mathcal{I}}_{2} & \equiv \int_{\mathrm{U}(N)} d \mu(U) \int_{\mathrm{U}(N)} d \mu(V) \operatorname{det}^{\nu}(U A V B) e^{\operatorname{tr}\left(U A V B+C V^{\dagger} D U^{\dagger}\right)} \\
& =\sum_{r} \sum_{r^{\prime}} \alpha_{r}^{(\nu)} \alpha_{r^{\prime}}^{(0)} \int_{\mathrm{U}(N)} d \mu(U) \int_{\mathrm{U}(N)} d \mu(V) \chi_{r}(U A V B) \chi_{r^{\prime}}\left(C V^{\dagger} D U^{\dagger}\right) \\
& =\sum_{r} \frac{\alpha_{r}^{(\nu)} \alpha_{r}^{(0)}}{d_{r}} \int_{\mathrm{U}(N)} d \mu(U) \chi_{r}\left(B U A D U^{\dagger} C\right) \\
& =\sum_{r} \frac{\alpha_{r}^{(\nu)} \alpha_{r}^{(0)}}{d_{r}^{2}} \chi_{r}(A D) \chi_{r}(B C),
\end{aligned}
$$

where we have made use of Eqs. (8) and (9). From Eq. (11) we have

$$
\frac{\alpha_{r}^{(\nu)} \alpha_{r}^{(0)}}{d_{r}^{2}}=\left[\prod_{n=1}^{N-1} n !\right]^{2} \prod_{i=1}^{N} \frac{1}{k_{i} !\left(k_{i}-\nu\right) !} .
$$


Now denote the eigenvalues of the matrices $A D$ and $B C$ by $x_{1}^{2}, \ldots, x_{N}^{2}$ and $y_{1}^{2}, \ldots, y_{N}^{2}$, respectively. Using again Weyl's formula (12), Eq. (21) thus becomes

$$
\hat{\mathcal{I}}_{2}=\left[\prod_{n=1}^{N-1} n !\right]^{2} \frac{1}{\Delta\left(x^{2}\right) \Delta\left(y^{2}\right)} \sum_{r} \prod_{i=1}^{N} \frac{1}{k_{i} !\left(k_{i}-\nu\right) !} \operatorname{det}\left[x_{i}^{2 k_{j}}\right] \operatorname{det}\left[y_{i}^{2 k_{j}}\right] .
$$

Employing the expansion theorem given in Eq. (B2) of Ref. [14] and noting Eq. (15) again, this yields

$$
\hat{\mathcal{I}}_{2}=\left[\prod_{n=1}^{N-1} n !\right]^{2} \frac{1}{\Delta\left(x^{2}\right) \Delta\left(y^{2}\right)} \operatorname{det}\left[f\left(x_{i} y_{j}\right)\right]
$$

with

$$
f(z)=z^{\nu} I_{-\nu}(2 z) .
$$

Pulling the factors of $x_{i}^{\nu}$ and $y_{j}^{\nu}$ out of the determinant and using $I_{n}=I_{-n}$, we obtain

$$
\hat{\mathcal{I}}_{2}=\left[\prod_{n=1}^{N-1} n !\right]^{2} \operatorname{det}^{\frac{\nu}{2}}(A B C D) \frac{\operatorname{det}\left[I_{\nu}\left(2 x_{i} y_{j}\right)\right]}{\Delta\left(x^{2}\right) \Delta\left(y^{2}\right)} .
$$

We finally rescale $A, B, C$ and $D$ by $1 / \sqrt{2}$ to reinstate the factor of $\frac{1}{2}$ in the exponent of Eq. (2) to obtain

$$
\begin{aligned}
\int_{\mathrm{U}(N)} d \mu(U) \int_{\mathrm{U}(N)} d \mu(V) \operatorname{det}^{\nu}(U V) e^{\frac{1}{2} \operatorname{tr}\left(U A V B+C V^{\dagger} D U^{\dagger}\right)} \\
=2^{N(N-1)}\left[\prod_{n=1}^{N-1} n !\right]^{2}\left(\frac{\operatorname{det}(C D)}{\operatorname{det}(A B)}\right)^{\frac{\nu}{2}} \frac{\operatorname{det}\left[I_{\nu}\left(x_{i} y_{j}\right)\right]}{\Delta\left(x^{2}\right) \Delta\left(y^{2}\right)} .
\end{aligned}
$$

Once again, the $x_{i}^{2}$ and $y_{j}^{2}$ are the eigenvalues of $A D$ and $B C$, respectively.

Let us now consider the case in which $U$ and $V$ have different dimensions, i.e.,

$$
\mathcal{I}_{2}^{(N, M)}=\int_{\mathrm{U}(N)} d \mu(U) \int_{\mathrm{U}(M)} d \mu(V) e^{\frac{1}{2} \operatorname{tr}\left(U A V B+C V^{\dagger} D U^{\dagger}\right)} .
$$

In this case, $A$ and $C$ are complex $N \times M$ matrices, and $B$ and $D$ are complex $M \times N$ matrices. For definiteness, we shall take $M<N$.

At this point, we have not been able to prove a result for $\mathcal{I}_{2}^{(N, M)}$, but we conjecture, based on explicit calculations for small $N$ and $M$, as well as on numerical experimentation, that the result takes the form

$$
\mathcal{I}_{2}^{(N, M)}=2^{M(N-1)}\left[\prod_{n=N-M}^{N-1} n !\right]\left[\prod_{m=N-M}^{M-1} m !\right] \frac{\operatorname{det}\left[I_{N-M}\left(x_{i} y_{j}\right)\right]}{\Delta\left(x^{2}\right) \Delta\left(y^{2}\right) \prod_{i=1}^{M}\left(x_{i} y_{i}\right)^{N-M}} .
$$

Here, $x_{i}^{2}$ and $y_{i}^{2}(i=1, \ldots, M)$ denote the (non-zero) eigenvalues of $D A$ and $B C$, respectively. This expression also reduces to the well-known result in the case where $C=B^{\dagger}$ and $D=A^{\dagger}$, cf. Refs. [15, 10, 16].

Note that we have not included any determinant terms in the integrand of $\mathcal{I}_{2}^{(N, M)}$. If we included, say, $\operatorname{det}^{\nu} U \operatorname{det}^{\eta} V$ in the integrand, integrations over the $\mathrm{U}(1)$ subgroups of $\mathrm{U}(N)$ and $\mathrm{U}(M)$ show immediately that the value of the resulting integral is zero unless, possibly, $\nu$ and $\eta$ are related by $N \nu=M \eta$. We now show that, even if this relation holds, the integral gives zero for any $\nu \neq 0$, and 
hence also for $\eta \neq 0$. To see this, suppose that $\nu \neq 0$, and let us perform the integral of $U$ over $\mathrm{U}(N)$, leaving the integral over $\mathrm{U}(M)$ untouched for the moment. The result could be read off from Eq. (18) if the matrices $A V B$ and $C V^{\dagger} D$ were $\operatorname{Gl}(N)$ matrices. However, since $M<N$, these matrices are not of full rank, and therefore $N-M$ of their eigenvalues are equal to zero. Except on a set of measure zero they have the same rank, so that a limiting process leads to a finite value of $\operatorname{det}\left(C V^{\dagger} D\right) / \operatorname{det}(A V B)$, given by $\operatorname{det}\left(V^{\dagger} D C\right) / \operatorname{det}(V B A)$. We then need to find the limit of $\operatorname{det}\left[\mu_{i}^{j-1} I_{\nu+j-1}\left(\mu_{i}\right)\right] / \Delta\left(\mu^{2}\right)$ as $\mu_{M+1}, \ldots, \mu_{N} \rightarrow 0$. In this context, $\mu_{1}^{2}, \ldots, \mu_{M}^{2}$ denote the non-zero eigenvalues of $A V B C V^{\dagger} D$. In fact, letting just $\mu_{N} \rightarrow 0$, it is easy to see that the above expression goes to zero, unless $\nu=0$, which shows that $\mathcal{I}_{2}^{(N, M)}=0$, unless $\nu=\eta=0$.

\subsection{Generalization of the Itzykson-Zuber integral}

The integral

$$
\mathcal{I}_{3}=\int_{\mathrm{U}(N)} d \mu(U) e^{\operatorname{tr}\left(A U B U^{\dagger}\right)}=\left[\prod_{n=1}^{N-1} n !\right] \frac{\operatorname{det}\left[\exp \left(x_{i} y_{j}\right)\right]}{\Delta(x) \Delta(y)}
$$

was computed in Ref. 18 for the case where $A$ and $B$ are hermitian matrices with real eigenvalues $x_{1}, \ldots, x_{N}$ and $y_{1}, \ldots, y_{N}$, respectively. This is a special case of a more general result due to Harish-Chandra [19].

Following the calculation of this integral in Ref. [14], it is immediately obvious that the only change in the final result is the replacement of the eigenvalues of the hermitian matrices $A$ and $B$ by the eigenvalues of their general complex versions.

Also, including the determinant of $U$ in the integrand (raised to any nonzero power) would give zero due to the integration over the U(1) subgroup.

\subsection{Comment on noninvertible matrices}

In deriving the above results, we have assumed that the matrices $A, B, C$ and $D$ are elements of $\operatorname{Gl}(N, \mathbb{C})$. However, the integrals $\mathcal{I}_{1}$ through $\mathcal{I}_{3}$ exist even if the matrices on which they depend are not of full rank. In this case, we can consider the limit in which one or more of the eigenvalues of the matrix approach zero. Uniform convergence permits the interchange of this limit and the integration over the unitary group. A l'Hôpital procedure on the right-hand side of Eq. (18), (27) or (30) then leads to a finite (though possibly zero) result.

\section{Conclusions}

We have derived generalizations of several well-known integrals over the unitary group to the case where general complex matrices appear in the integrand. These integrals may find applications in lattice gauge theory but are also of purely mathematical interest, in particular in the theory of random matrices. As mentioned in the introduction, our motivation for studying these integrals originated from the complex action problem that arises if the color-flavor transformation is applied to lattice QCD. We found that the integral $\mathcal{I}_{1}$ solved this complex action problem for one quark flavor but unfortunately not for two or more flavors. 
Our results were obtained by a straightforward application of Balantekin's character expansion method. It would be interesting to investigate the feasibility of other well-known methods to compute integrals over the unitary group, such as the diffusion equation method, in the cases we have considered. It should also be possible to generalize the results of the present paper to integrals over the super-unitary group.

\section{Acknowledgments}

This work was supported in part by the U.S. Department of Energy under contract No. DE-FG02-91ER40608 and in part under the ECT* 'STATE' contract. We thank T. Guhr for interesting conversations.

\section{References}

[1] J. Miller and J. Wang, Phys. Rev. Lett. 76 (1996) 1461; N. Hatano and D.R. Nelson, Phys. Rev. Lett. 77 (1996) 570.

[2] R. Grobe, F. Haake and H.-J. Sommers, Phys. Rev. Lett. 61 (1988) 1899; Y.V. Fyodorov and B.A. Khoruzhenko, Phys. Rev. Lett. 83 (1999) 65.

[3] M.A. Stephanov, Phys. Rev. Lett. 76 (1996) 4472.

[4] M.R. Zirnbauer, J. Phys. A 29 (1996) 7113.

[5] B. Schlittgen and T. Wettig, Nucl. Phys. B 632 (2002) 155.

[6] J. Budczies, S. Nonnenmacher_Y Y Shnir and_M.R. Zirnbauer, Nucl. Phys. B 635 (2002) 309.

[7] B. Schlittgen and T. Wettig, hep-lat/0208044.

[8] S. Nonnenmacher and Y. Shnir, hep-lat/0210002

[9] R.C. Brower, P. Rossi and C.-I Tan, Phys. Rev. D 23 (1981) 942.

[10] A.D. Jackson, M.K. Şener and J.J.M. Verbaarschot, Phys. Lett. B 387 (1996) 355.

[11] T. Akuzawa and M. Wadati, J. Phys. Soc. Jap. 67 (1998) 2151.

[12] H. Leutwyler and A. Smilga, Phys. Rev. D 46 (1992) 5607.

[13] B.A. Balantekin, J. Math. Phys. 25 (1984) 2028.

[14] B.A. Balantekin, Phys. Rev. D 62 (2000) 085017.

[15] F.A. Berezin and F.I. Karpelevich, Doklady Akad. Nauk SSSR 118 (1958) 9.

[16] T. Guhr and T. Wettig, J. Math. Phys. 37 (1996) 6395.

[17] L.K. Hua, Harmonic Analysis of Functions of Several Complex Variables in the Classical Domains (American Mathematical Society, Providence, Rhode Island, 1963).

[18] C. Itzykson and J.B. Zuber, J. Math. Phys. 21 (1980) 411.

[19] Harish-Chandra, Am. J. Phys. 80 (1958) 241. 